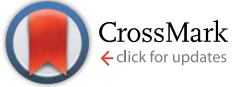

Cite this: RSC Adv., 2017, 7, 2810

Received 19th October 2016 Accepted 2nd December 2016

DOI: $10.1039 / c 6 r a 25465 a$

www.rsc.org/advances

\section{Surface passivation of carbon dots with ethylene glycol and their high-sensitivity to $\mathrm{Fe}^{3+} \uparrow$}

\begin{abstract}
Zhaogan Wang,,$^{\mathrm{a}}$ Peng Long, $\dot{\hbar}^{\mathrm{a}}$ Yiyu Feng, ${ }^{\text {acd }}$ Chengqun Qin $^{\mathrm{a}}$ and Wei Feng ${ }^{\star a b c d}$
Hydroxyl functionalized carbon dots ( $\mathrm{H}-\mathrm{CDs}$ ) were prepared by monoesterification of ethylene glycol. The $\mathrm{H}$-CDs exhibit a narrow size distribution of 1-4 $\mathrm{nm}$ and enhanced photoluminescent (PL) intensity due to an increased amount of electron donor hydroxyl groups. According to fluorescence spectra, the $\mathrm{H}$-CDs exhibit a high sensitivity to $\mathrm{Fe}^{3+}$ with a detection limit of $2.56 \mathrm{nM}$, which is superior to the detection limit of CDs $(7.4 \mu \mathrm{M})$. The quenching fluorescence is primarily controlled by the formation of a chelate compound based on the complexation between $\mathrm{Fe}^{3+}$ and the hydroxyl on the surface of the $\mathrm{H}$-CDs. Furthermore, we demonstrate that paper impregnated with $\mathrm{H}$-CDs exhibits a high sensitivity to $\mathrm{Fe}^{3+}$ by fluorescence quenching. In the future, the modified CDs can be developed for high sensitivity fluorescent probes by optimizing the chemical structures and microstructures.
\end{abstract}

\section{Introduction}

Iron(III) ion is an essential element of hemoglobin. It is vitally important for the environment to develop efficient detection technologies with high selectivity and sensitivity toward $\mathrm{Fe}^{3+}{ }^{1}$ Over the past several years, a large number of $\mathrm{Fe}^{3+}$ detection technologies have been rapidly developed, ${ }^{2}$ including flame atomic absorption spectroscopy (FAAS), atomic emission spectroscopy (AES), inductively coupled plasma mass spectrometry (ICPMS), and electrochemical sensors.

Recently, carbon dots (CDs) have become an attractive candidate as fluorescent probes for $\mathrm{Fe}^{3+}$ detection because of their outstanding physical, chemical, and optical properties (e.g., high photostability, water solubility, good biocompatibility, eco-friendly preparation, and widely available precursors). ${ }^{3-9}$ In the past few years, significant efforts have been devoted to the synthesis of fluorescent carbon dots for $\mathrm{Fe}^{3+}$ detection by many different research groups. Qu et al. first prepared fluorescent CDs by using a simple hydrothermal

${ }^{a}$ School of Materials Science and Engineering, Tianjin University, Tianjin 300072, P. R. China.E-mail:weifeng@tju.edu.cn

${ }^{b}$ Collaborative Innovation Center of Chemical Science and Engineering (Tianjin), Tianjin 300072, P. R. China

${ }^{c}$ Key Laboratory of Advanced Ceramics and Machining Technology, Ministry of Education, Tianjin 300072, P. R. China

${ }^{d}$ Tianjin Key Laboratory of Composite and Functional Materials, Tianjin 300072, P. R. China

$\dagger$ Electronic supplementary information (ESI) available: The fluorescence spectrum of CDs and H-CDs at different concentrations, the PL spectra of the CDs solutions in the presence of $\mathrm{Fe}^{3+}$ when excited at different wavelength, the Stern-Volmer relationship of CDs between the PL intensity and the concentration, the standard deviations of the detection limit and selectivity for H-CDs. See DOI: 10.1039/c6ra25465a

\$ Contributed equally. treatment on dopamine; the resulting CDs could serve as $\mathrm{Fe}^{3+}$ sensors with a detection limit as low as $0.32 \mu \mathrm{M}^{8}{ }^{8}$ Chen et al. presented a facile electrochemical strategy for graphene quantum dots (GQDs) from monolithic 3D graphene. The GQDs show an optical selectively and sensitivity towards $\mathrm{Fe}^{3+}$ with a detection limit of $7.22 \mu \mathrm{M} .{ }^{9}$ However, the CDs or GQDs, without surface-passivation, possess multiple disadvantages, including an unsatisfactory $\mathrm{Fe}^{3+}$ detection limit and a relatively low quantum yield. ${ }^{10}$

Previous studies showed that the oxygen-containing functional groups of CDs play an important role in the recombination or separation of electron-hole pairs, which affects the fluorescence properties. ${ }^{11-13}$ Specifically, the carboxyl groups can form nonradiative recombination centres that act as electron acceptors, thus lowering fluorescence. ${ }^{\mathbf{1 4 - 1 6}}$ In contrast, the hydroxyl groups, which act as electron donors, facilitate fast carrier mobility, which would be of benefit to the fluorescence emission. ${ }^{12}$ Thus, the surface chemistry of the CDs is a key parameter for the luminescence performance..$^{15,17,18}$ According to previous studies, the hydroxyls on the surface of CDs form complexes with $\mathrm{Fe}^{3+}$, owing to coordination interaction. ${ }^{19}$ The formed Fe-CDs complexes could facilitate charge transfer and restrain exciton recombination, leading to significant fluorescence quenching. ${ }^{\mathbf{2 0 , 2 1}}$

Thus, in order to improve the fluorescence intensity and the detection sensitivity, we report a simple synthetic route to prepare hydroxyl functionalized fluorescent carbon dots. In this experiment, we utilize the hydroxyl group of ethylene glycol and the carboxyl on the surface of the CDs to synthesize hydroxyl functionalized fluorescent carbon dots through monoesterification. The schematic illustration of the synthesis of the CDs and the detection mechanism are shown in Scheme 1. First, the CDs for hydroxyl functionalized were synthesized via 
(a)

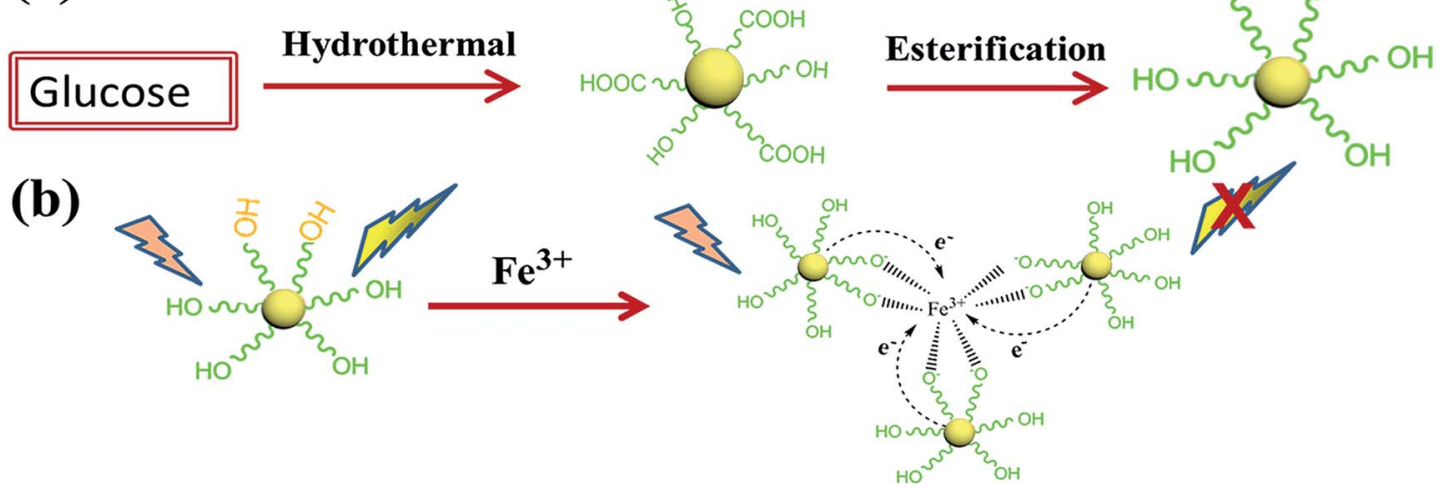

Scheme 1 A schematic illustration of (a) the synthesis procedure of the $\mathrm{H}-\mathrm{CDs}$ and (b) the formation process between $\mathrm{H}$ - $\mathrm{CDs}$ and Fe ${ }^{3+}$ for the chelate compound.

a hydrothermal reaction. Then, we chose $\mathrm{MeSO}_{3} \mathrm{H}$ and $\mathrm{Al}_{2} \mathrm{O}_{3}$ as catalytic agents to synthesize hydroxyl functionalized carbon dots (H-CDs) through monoesterification with ethylene glycol. The microstructures of the CDs and H-CDs were characterized by high-resolution transmission electron microscopy (HRTEM). Fourier transform infrared (FTIR) spectroscopy and X-ray photoelectron spectroscopy (XPS) were used to measure the chemical structures of the CDs and H-CDs. The optical properties were investigated by UV-vis absorption and photoluminescence (PL) spectra. Furthermore, we demonstrate the proof of concept that CDs with hydroxyls covered surfaces can be used as a very effective fluorescent sensing platform for selective detection of $\mathrm{Fe}^{3+}$.

\section{Experimental}

\subsection{Materials}

Glucose and $\mathrm{MeSO}_{3} \mathrm{H}$ were obtained from Aladdin chemistry Co. Ltd. (Shanghai, China), $\mathrm{Al}_{2} \mathrm{Cl}_{3}, \mathrm{ZnCl}_{2}, \mathrm{BaCl}_{2}, \mathrm{FeCl}_{2}, \mathrm{CuCl}_{2}$, $\mathrm{NiCl}_{3}, \mathrm{CrCl}_{3}, \mathrm{PbCl}_{2}, \mathrm{CoCl}_{2}, \mathrm{FeCl}_{3}$ and $\mathrm{AgNO}_{3}$ were purchased from J\&K Chemical Company (Beijing, China). Anhydrous ethanol and deionized water were obtained from Tianjin Guangfu technology development Co., Ltd. All the chemicals were analytical grade reagents and used without further purification.

\subsection{Preparation of CDs}

The initial CDs for hydroxyl functionalization were synthesized via a hydrothermal reaction, together with a mount of reagent for the formation of the CDs. In a typical experiment, $1000 \mathrm{mg}$ of glucose was dispersed in $80 \mathrm{ml}$ of ethanol by sonication for 10 min using an ultrasonic generator. Next, the mixture was transferred into a $150 \mathrm{ml}$ Teflon lined autoclave and heated at $180^{\circ} \mathrm{C}$ for $18 \mathrm{~h}$. After that, the products were then allowed to cool to ambient temperature naturally. The obtained solution was collected by removing the impurities through centrifugation at $16000 \mathrm{rpm}$ for $15 \mathrm{~min}$.

\subsection{General procedure for $\mathrm{H}-\mathrm{CDs}$}

In a typical experiment, $\mathrm{MeSO}_{3} \mathrm{H}(1 \mathrm{ml}, 15 \mathrm{mmol})$ and $\mathrm{Al}_{2} \mathrm{O}_{3}$ ( $270 \mathrm{mg}, 3 \mathrm{mmol}$ ), which were the catalytic agents, were added to the above CDs $(20 \mathrm{ml})$. The mixture was stirred and heated at $80{ }^{\circ} \mathrm{C}$ for 8 h. ${ }^{22}$ Next, the solution was centrifuged at $16000 \mathrm{rpm}$ for $15 \mathrm{~min}$ to separate out the catalytic agents. Finally, the obtained H-CDs were dialyzed in water through a dialysis membrane (500 MWCO) for $72 \mathrm{~h}$.

\subsection{Fluorescent sensing of $\mathrm{Fe}^{3+}$}

In a typical assay, $1.00 \mathrm{ml}$ of the CDs solution $\left(1 \mathrm{mg} \mathrm{m}^{-1}\right)$, which were dispersed in deionized water, was placed in a $10 \mathrm{ml}$ colorimetric tube, and $1.00 \mathrm{ml}$ of a phosphate buffer saline (PBS, $10 \mathrm{mM}, \mathrm{pH}=7$ ) solution was added to keep the system under a neutral $\mathrm{pH}$ condition. Following this, different concentrations of $\mathrm{Fe}^{3+}$ solution $(10 \mu \mathrm{l})$, which were obtained by dispersing $\mathrm{FeCl}_{3}$ in deionized water, were added. The FL spectra were recorded after reaction for $5 \mathrm{~min}$. The selectivity of $\mathrm{Fe}^{3+}$ was confirmed by adding other metal ions solutions $\left(\mathrm{Al}_{2} \mathrm{Cl}_{3}\right.$, $\mathrm{ZnCl}_{2}, \mathrm{BaCl}_{2}, \mathrm{FeCl}_{2}, \mathrm{CuCl}_{2}, \mathrm{NiCl}_{3}, \mathrm{CrCl}_{3}, \mathrm{PbCl}_{2}, \mathrm{CoCl}_{2}$ and $\mathrm{AgNO}_{3}$ ) in place of $\mathrm{FeCl}_{3}$ in a similar way. All experiments were performed at room temperature.

\subsection{Determination of fluorescence quantum yield}

The quantum yield (QY) of H-CDs was calculated according to a previously reported procedure. ${ }^{23,24}$ The optical densities were obtained on a UV-vis spectrophotometer. Quinine sulfate was dissolved in $0.1 \mathrm{M} \mathrm{H}_{2} \mathrm{SO}_{4}$ as a reference standard. Absolute values were measured using the standard reference sample, which has a fixed fluorescence quantum yield value (0.54 at 360 $\mathrm{nm})$. The QY of the CDs was calculated by the following equation with five different concentrations.

$$
Q_{\mathrm{s}}=Q_{\mathrm{R}}\left(K / K_{\mathrm{R}}\right)\left(\eta / \eta_{\mathrm{R}}\right)^{2}
$$

where the $Q$ is the quantum yields, $K$ is the slope determined by the curves, and $\eta$ is the refractive index of the solvent. ${ }^{25}$ For these aqueous solutions, the refractive index for the solvents is 
1.33. The subscript " $R$ " refers to the standard with known quantum yields. In order to minimize re-absorption effects, the absorbance in a $10 \mathrm{~mm}$ fluorescence cuvette should not exceed 0.1 absorbance units during the UV-vis spectrum measurement.

\subsection{Characterization}

The microstructures of the CDs and H-CDs were recorded by TEM (Philips Tecnai G2F20). FTIR spectra were obtained on Bruker Tensor 27 spectrometer, XPS analysis was completed with a PERKIN ELMZR PHI 3056 spectrometer with an $\mathrm{Al}$ anode source operated at $15 \mathrm{kV}$ and an applied power of $350 \mathrm{~W}$. UV-vis absorption and PL were studied using a spectrophotometer (UV-3600, Shimadzu) and a spectrofluorometer (Fluorolog3, HORIBA, Jobin Yvon), respectively.

\section{Results and discussion}

The oxygenic functional groups in the glucose molecule and the high temperature and pressure conditions make it easy for a dehydration reaction to occur during the hydrothermal reaction. Via the dehydration reaction, the glucose molecules were pyrolyzed, cross-linked, and finally converted into carbon dots. $^{26,27}$ Various oxygenic groups were introduced along the surface of the carbon dots in the hydrothermal reaction process. Normally, glucose cannot dissolve in ethanol; however, through the hydrothermal reaction process, the glucose is dissolved in the solution and a change in colour occurs (e.g., from transparent to dark brown) as a result of the formation of CDs. Moreover, after modification, the colour of CDs turns from dark brown to yellow. This is because the carboxyl moieties on the CDs changed to hydroxyl groups and thus significantly increased the amount of hydroxyl groups on the surface of CDs.

Fig. 1 shows the TEM images of the CDs and H-CDs. The prepared CDs and H-CDs were mostly spherical in shape and monodispersed without agglomeration. The distributions of particle sizes are shown in the insets, indicating that the carbon dots was uniformly distributed. It is worth noting that the $\mathrm{H}$ CDs exhibit a narrower size distribution. As shown in these figures, the diameters of the H-CDs are in the range of 1-3 nm (average of $2.0 \mathrm{~nm}$ ), which is smaller than CDs (average of 3.5 $\mathrm{nm})$. This feature is attributed to the etching of CDs surface by
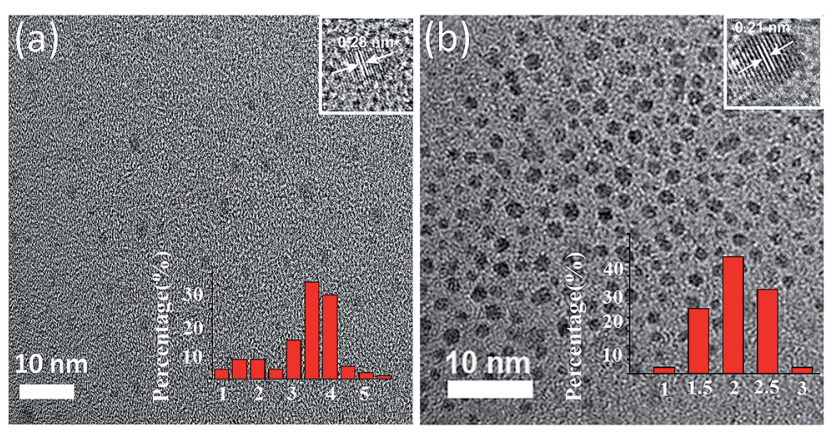

Fig. 1 TEM images of (a) the CDs and (b) H-CDs, the top insets are HRTEM images of these CDs and the lower insets show the size distribution calculated based on 50 nanoparticles. the ligand. ${ }^{28-30}$ HRTEM images (insets) of CDs and H-CDs show well-resolved lattice fringes with an in-plane lattice spacing of $0.28 \mathrm{~nm}$ and $0.21 \mathrm{~nm}$, respectively, which are consistent with the (020), (100) diffraction facets of graphite carbon, respectively. ${ }^{31,32}$

Fig. 2 shows the FTIR and the XPS data of the CDs and H-CDs. From the FT-IR spectra of the CDs, the obvious absorption peak centred at $1652 \mathrm{~cm}^{-1}$, which is caused by $\mathrm{C}=\mathrm{C}$ stretching vibration, can be observed. Moreover, the bending vibration of $\mathrm{C}-\mathrm{H}$ at $937 \mathrm{~cm}^{-1}$ and $2965 \mathrm{~cm}^{-1}$ further confirmed the existence of $\mathrm{C}=\mathrm{C}$. During the hydrothermal reaction process, the precursor molecules were dehydrated to form $\mathrm{C}=\mathrm{C}$ which is the elementary unit of the as-prepared CDs. ${ }^{26}$ From the FT-IR spectra of H-CDs, an obvious absorption peak at $1719 \mathrm{~cm}^{-1}$, which is caused by $\mathrm{C}=\mathrm{O}$ stretching vibration, can be observed. In Fig. 2a, the peaks at $1197 \mathrm{~cm}^{-1}$ and $1057 \mathrm{~cm}^{-1}$ are ascribed to the stretching vibrations of the ester group on the surface of $\mathrm{H}$ CDs. This feature is indicative of the change from $-\mathrm{COOH}$ to -OH by an esterification reaction on the surface of H-CDs.

To further confirm the type of functional groups on the surfaces of the as-prepared CDs and H-CDs, XPS characterization was carried out. As shown in Fig. 2b, the CDs only show $\mathrm{C}$ and $\mathrm{O}$ signals. This implies that the carbon dots contained only oxygenic groups. The C1s spectra of the as-prepared CDs and $\mathrm{H}-$ CDs are shown in Fig. $2 c$ and d, respectively. The measured spectra of the "pure" CDs (Fig. 2c) can be deconvoluted into five surface components, corresponding to $\mathrm{sp}^{2}(\mathrm{C}=\mathrm{C})$ at the binding energy of $284.5 \mathrm{eV}, \mathrm{sp}^{3}(\mathrm{C}-\mathrm{C}$ and $\mathrm{C}-\mathrm{H})$ at $285.5 \mathrm{eV}, \mathrm{C}-$ $\mathrm{OH}$ at $286.6 \mathrm{eV}, \mathrm{C}-\mathrm{O}-\mathrm{C}$ at $287.2 \mathrm{eV}$, and $\mathrm{C}=\mathrm{O}$ at $288.6 \mathrm{eV} \cdot{ }^{33}$ The intensity of $\mathrm{C}-\mathrm{OH}$ for the $\mathrm{H}-\mathrm{CDs}$ shows a significant increase compared with the CDs. This feature arises from the increasing number of hydroxyl groups on the surface of the CDs. The XPS results imply that the amounts of oxygen-groups present on the surface of the CDs and H-CDs were different. These results determined by the XPS are in good agreement with FTIR results. These demonstrate that the hydroxyl of ethylene glycol has already grafted onto the surface carboxyl of the CDs.

Optical properties were investigated by UV-vis absorption and photoluminescence (PL) spectra. Ultraviolet-visible (UV) absorption spectrum results (Fig. 3a) show that the CDs and H-CDs exhibit two deep UV absorption peaks. The absorption peaks at 222 and $207 \mathrm{~nm}$ for the CDs and H-CDs, respectively, resulted from the $\pi-\pi^{*}$ transition of $\mathrm{C}=\mathrm{C}$, and the absorptions at 280 and $272 \mathrm{~nm}$ for the CDs and H-CDs, respectively, correspond to the $n-\pi^{*}$ transition of the $\mathrm{C}=\mathrm{O}$ bond..$^{\mathbf{3 4 3 5}}$ The observed deviations in the UV absorption indicate that the different kinds and amounts of the oxygen-containing groups can affect the optical properties of CDs. ${ }^{3}$

The PL emission spectra were primarily investigated under excitation wavelengths. The PL emission spectra show that both the CDs and H-CDs exhibit the strongest PL emission peak when excited at $360 \mathrm{~nm}$. Fig. $3 \mathrm{~b}$ and $\mathrm{c}$ indicate that the emission of the CDs and H-CDs are highly dependent on the excitation energy and the emission red-shifts with increasing excitation wavelength. Moreover, the PL intensities (Fig. 3d and S1†) of the $\mathrm{H}$-CDs are greatly enhanced in comparison with the CDs by reducing the amount of nonradiative recombination centres 

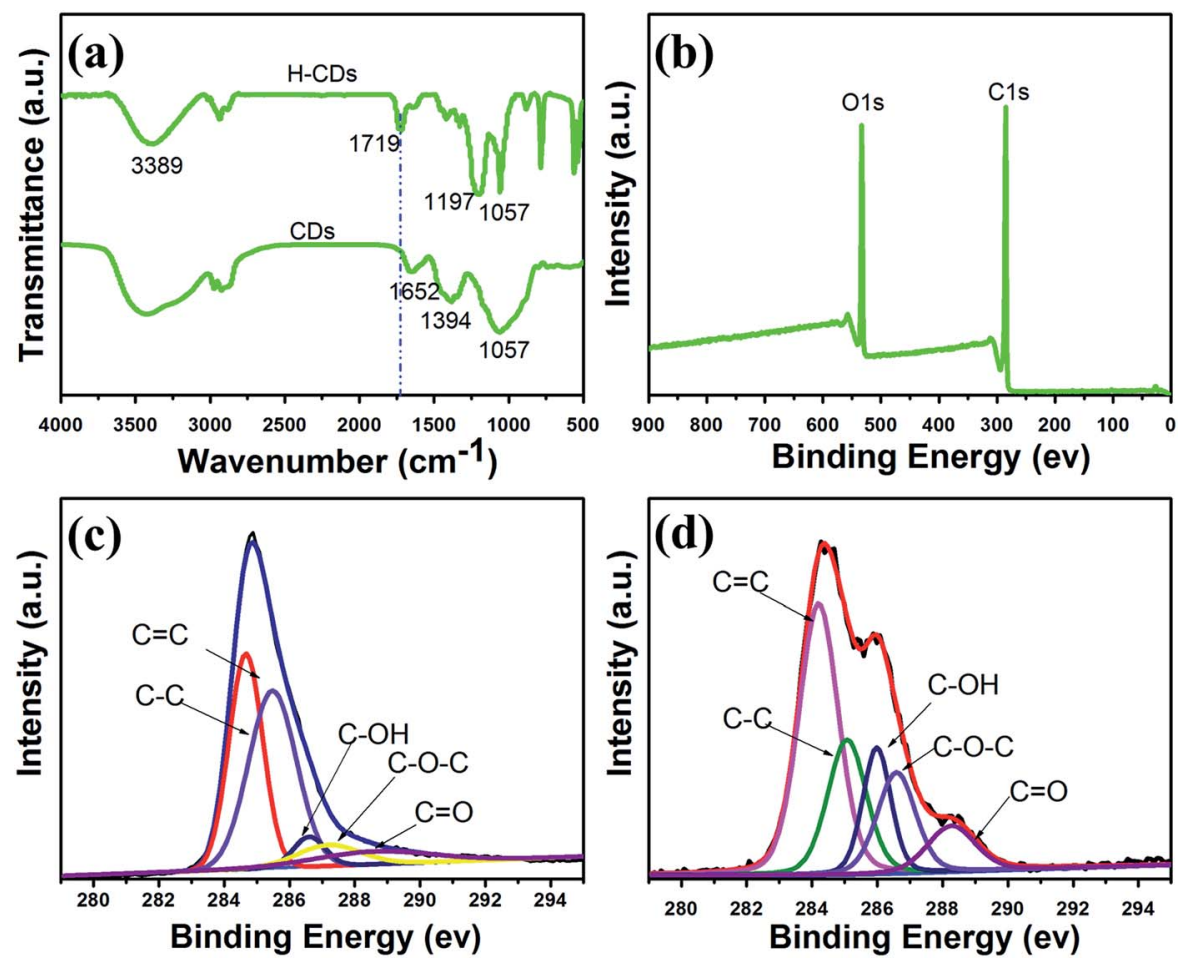

Fig. 2 FTIR spectra (a) of CDs and H-CDs; the full XPS spectrum (b) of CDs; the C1s XPS spectrum of CDs (c) and H-CDs (d).
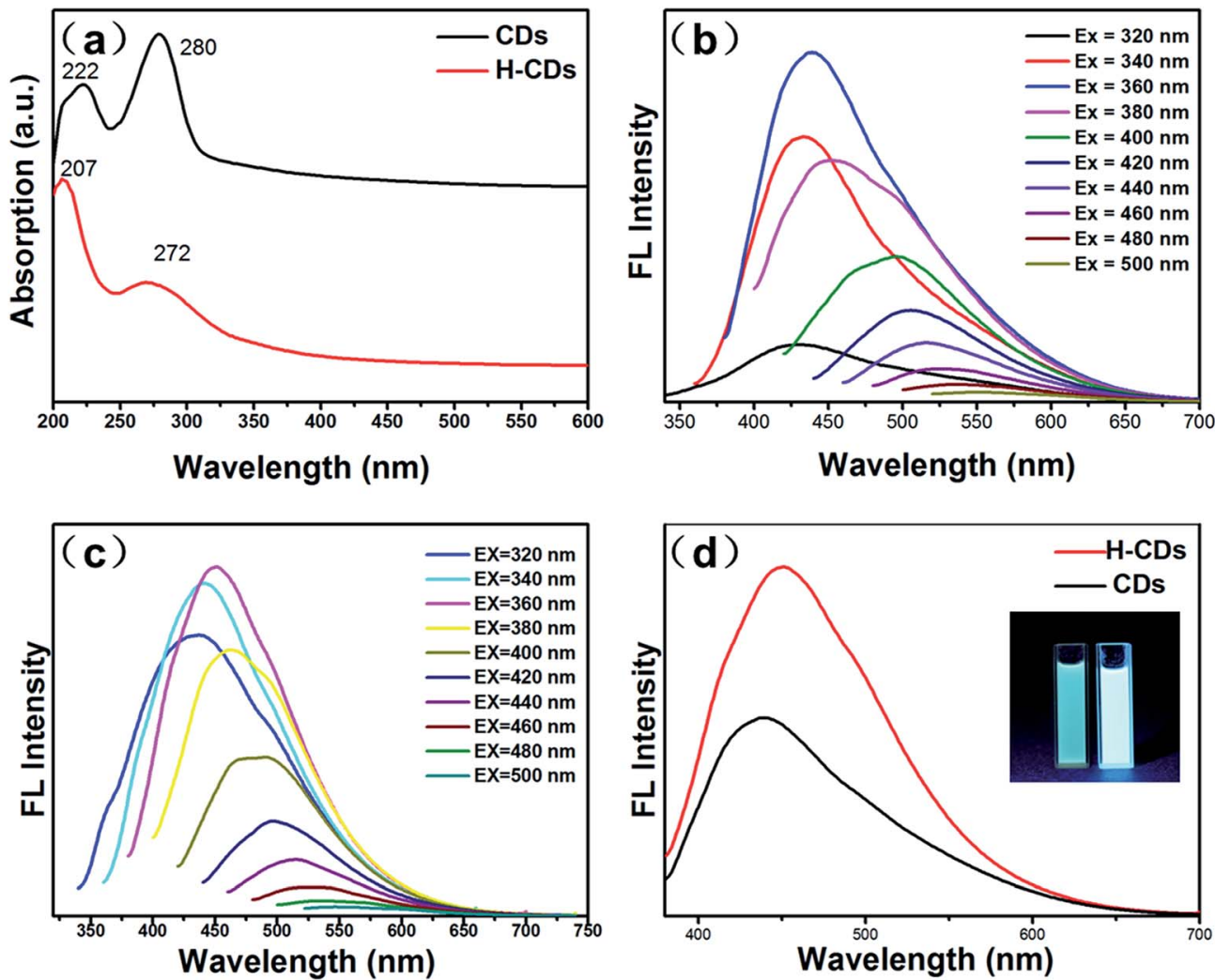

Fig. 3 (a) UV absorption of CDs and H-CDs, photoluminescence spectra of (b) CDs and (c) H-CDs from $320 \mathrm{~nm}$ to $500 \mathrm{~nm}$ excitation with a $20 \mathrm{~nm}$ increase in each step, (d) the fluorescence spectrum of CDs and H-CDs ( $\lambda_{\text {ex }}=360 \mathrm{~nm}$ ), The inset is a photograph of CDs (left) and HCDs (right) in aqueous solutions when excited at $360 \mathrm{~nm}$. 
caused by the carboxyl groups and increasing the amount of the hydroxyl groups, which contribute to enhancing the fluorescence intensity. ${ }^{3}$

It is worth pointing out that these as-prepared CDs also exhibit considerable photostability. No obvious aggregation could be observed even after storage at room temperature for several months, which is attributed to the electrostatic repulsions between the CDs resulting in electrostatic stabilization of the "pure" CDs and H-CDs in aqueous solution. The QYs of the H-CDs is $13 \%$ which were calculated according to the abovementioned rules, which is higher than that of the CDs (7\%). This indicates that the increase in surface hydroxyls on the CDs can improve the QYs.

The FTIR spectroscopy and XPS results show that we successfully synthesized H-CDs terminated with hydroxyl groups on the surface using the reaction described above. According to previous studies, hydroxyls on the surface of CDs could form complexes with $\mathrm{Fe}^{3+}$ ions owing to coordination interaction. ${ }^{19}$ During the complexation process, electrons in the excited state of the $\mathrm{H}$-CDs can transfer to the unfilled $\mathrm{Fe}^{3+}$ orbital, leading to nonradiative electron/hole recombination which results in fluorescence quenching. ${ }^{36}$ Therefore, we further investigated the PL spectra of the H-CDs and CDs solutions in the presence of $\mathrm{Fe}^{3+}$ at different concentrations.
Fig. 4a and $\mathrm{S} 2 \uparrow$ reveal that the PL intensities of the CDs solution are sensitive to $\mathrm{Fe}^{3+}$ concentration and decrease with an increase in $\mathrm{Fe}^{3+}$ concentration. Fig. $4 \mathrm{~b}$ and $\mathrm{S} 3 \dagger$ show the dependence of $\mathrm{FL}$ intensity on $\mathrm{Fe}^{3+}$ ions concentrations. Here, $\left(I_{0}-I\right) / I_{0}$ indicates a decrease in the FL intensity, where $I$ and $I_{0}$ represent the FL intensities of CDs at $360 \mathrm{~nm}$ in the presence and absence of $\mathrm{Fe}^{3+}$, respectively. The relationship between the concentration of $\mathrm{Fe}^{3+}$ and the PL intensities of CDs and H-CDs can be well fitted to the Stern-Volmer equation: $I_{0} / I=1+K_{\mathrm{sv}}[\mathrm{Q}]$, where [Q] is the concentration of $\mathrm{Fe}^{3+}$ and $K_{\mathrm{sv}}$ is the SternVolmer constant. ${ }^{37}$

The Stern-Volmer quenching curves (the inset of Fig. $4 \mathrm{~b}$ and $\mathrm{S} 3 \dagger$ ) show a good linearity with $K_{\mathrm{sv}}=1.9147787 \times 10^{7} \mathrm{M}^{-1}$ and $6.107 \times 10^{3} \mathrm{M}^{-1}$, indicating that the dynamic quenching processes occur in this sensor system. Moreover, the detection limit for $\mathrm{H}-\mathrm{CDs}$ is estimated to be $2.56 \mathrm{nM}$ based on $3 S_{\mathrm{b}} / k$ (here $S_{\mathrm{b}}$ was the standard deviation of the blank for 11 replicate measurements and $k$ was the slope of the calibration curve in the linear range), which is superior to the detection limit of the pure CDs $(7.4 \mu \mathrm{M})$. In order to guarantee the accuracy of our experimental results for the detection limit, we have repeated 11 times of experiments with the concentration of $\mathrm{Fe}^{3+}$ range from 0-100 nM. We calculated the detection limit of $\mathrm{H}$-CDs for $\mathrm{Fe}^{3+}$ in these repeated experiments, respectively. And then the
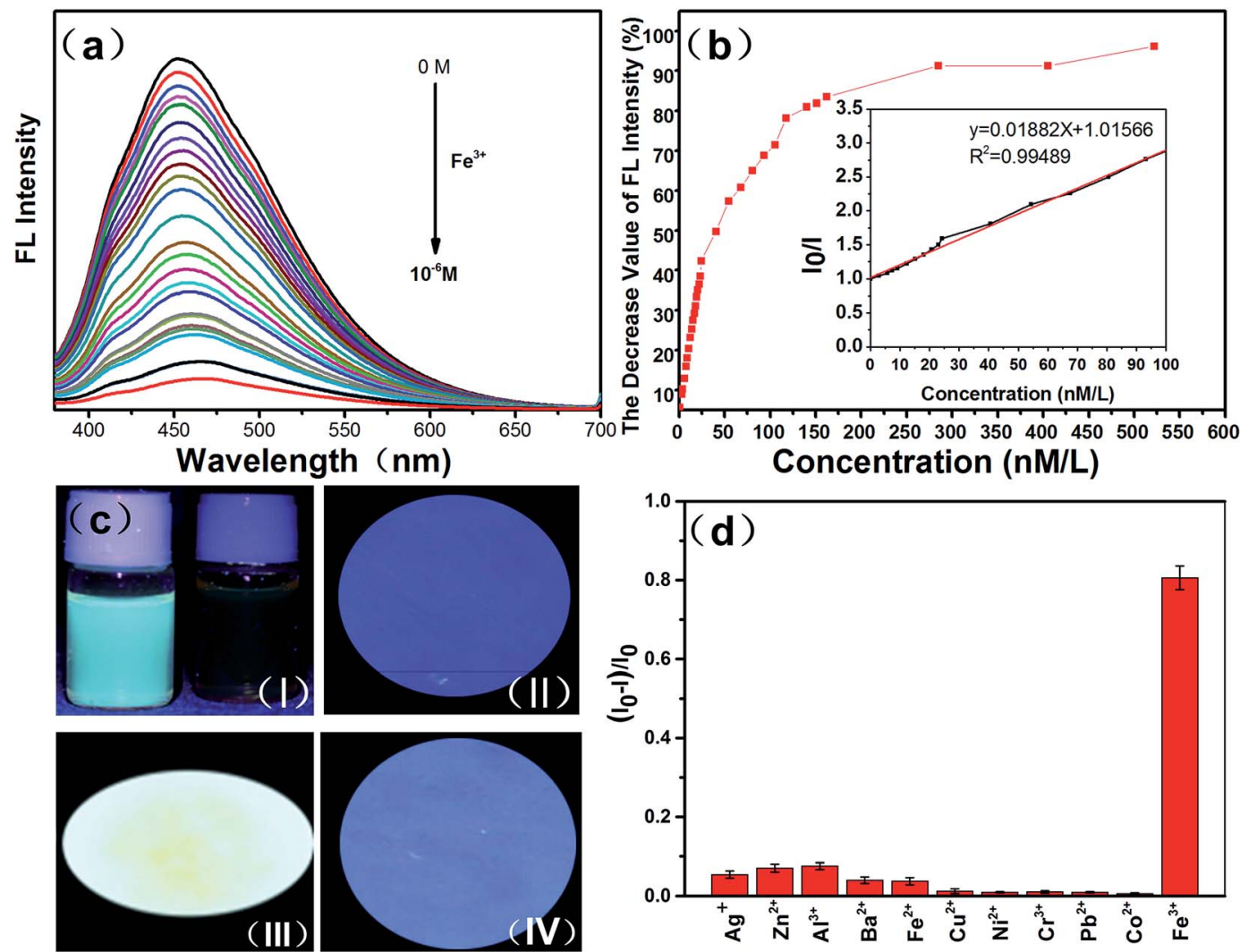

Fig. 4 (a) $\mathrm{PL}$ intensity of $\mathrm{H}$-CDs in the presence of different concentrations of $\mathrm{Fe}^{3+}$; (b) Stern-Volmer relationship between the $\mathrm{PL}$ intensity and the concentration. The inset displays a linear Stern-Volmer plot over a low Fe ${ }^{3+}$ concentration range; (c) photographs of (I) the $\mathrm{H}$ - $\mathrm{CDs}$ solution in the presence (right) and absence (left) of $\mathrm{Fe}^{3+}$, the filter paper under $360 \mathrm{~nm}$ lamp without any treated (II) and after immersion in the solution of $\mathrm{H}$-CDs in the absence (III) and presence (IV) of $\mathrm{Fe}^{3+}$, respectively; (d) the ratio of decrease in PL intensity for $\mathrm{H}-\mathrm{CDs}$ with the addition of different metal ions. 
standard deviation of these detection limits was figured out. The detection limits for these experiments were shown in Table S1. $\dagger$ After calculation, the standard deviation of the detection limit for H-CDs is $0.0773 \mathrm{nM}$. These indicate that the H-CDs show a great promise in the field of detecting $\mathrm{Fe}^{3+}$.

Li $e t$ al. have reported that the fluorescence of sulfur-doped graphene quantum dots (S-GQDs) could be effectively quenched by $\mathrm{Fe}^{3+}$ with a detection limit of $4.2 \mathrm{nM}^{38}$ Doping with $\mathrm{S}$ can effectively modulates the electronic local density of GQDs, which contribute to the coordination interaction between $\mathrm{Fe}^{3+}$ and hydroxyl groups on the edge of S-GQDs thus leading to significant fluorescence quenching. In this paper, we hold that the carboxyl groups can form nonradiative recombination centres that act as electron acceptors, thus lowering fluorescence, whereas the hydroxyl groups act as electron donors, facilitating fast carrier mobility and increasing fluorescence. Furthermore, the hydroxyls on the surface of CDs form the complexes with $\mathrm{Fe}^{3+}$ owing to the coordination interactions. The hydroxyls on the surface of CDs have a significant influence on the interaction with $\mathrm{Fe}^{3+}$. Thus, we utilize ethylene glycol to react chemically with the carboxyl groups of the CDs through glycol monoesters. In this way, we can reduce the content of carboxyl groups and increase the amount of hydroxyls on the surface of H-CDs. This not only enhanced the fluorescence intensity of the H-CDs but can also increase the number of reaction sites with $\mathrm{Fe}^{3+}$. The formed $\mathrm{Fe}-\mathrm{CDs}$ complexes could facilitate charge transfer and restrain exciton recombination, leading to significant fluorescence quenching. The increased reaction sites with $\mathrm{Fe}^{3+}$ resulting in an increase of the limit of detection. The remarkable fluorescence quenching of the $\mathrm{H}$-CDs shows great promise in detecting $\mathrm{Fe}^{3+}$. The complex formation is illustrated in Scheme 1. Furthermore, the $\mathrm{H}$-CDs can also be used in paper-based detection of $\mathrm{Fe}^{3+}$ as shown in Fig. 4c. First, the filter paper was immersed in the solution of H-CDs with a concentration of $1 \mathrm{mg} \mathrm{ml}^{-1}$. After $6 \mathrm{~h}$, the paper was taken out of the H-CDs solution and then dried in air at room temperature. We found that the H-CDs in filter paper can still maintain a good fluorescence (Fig. 4cIII). Moreover, when we added a few drops of $\mathrm{Fe}^{3+}$ to the filter paper, the fluorescence of the filter paper disappeared (Fig. 4cIV). This shows that the H-CDs can also shows a high sensitivity toward $\mathrm{Fe}^{3+}$ even in solid form.

To evaluate the sensitivity of H-CDs toward other metal ions, we further examined the PL intensity changes upon addition of other metal ions, including $\mathrm{Ag}^{+}, \mathrm{Zn}^{2+}, \mathrm{Al}^{3+}, \mathrm{Ba}^{2+}, \mathrm{Fe}^{2+}, \mathrm{Cu}^{2+}, \mathrm{Ni}^{2+}$, $\mathrm{Cr}^{3+}, \mathrm{Pb}^{2+}, \mathrm{Co}^{2+}$, under the same conditions. As is shown in Fig. 4d, no tremendous decrease in PL intensity was observed for $\mathrm{H}-\mathrm{CDs}$ in the presence of the above metal ions (1 mM) except for $\mathrm{Fe}^{3+}$. The concrete data of error bars for the selectivity of $\mathrm{H}-$ CDs was shown in Table S2. $\dagger$ The data of error bars are the standard deviation of the decrease value FL intensity during the repeated 11 times experiments. This indicates that the H-CDs have higher selectivity and sensitivity for $\mathrm{Fe}^{3+}$, whereas the other metal ions have little influence on the sensing system. Thus, the H-CDs could be applied in the field to the determine the presence of $\mathrm{Fe}^{3+}$. The reason why the $\mathrm{H}-\mathrm{CDs}$ are so sensitive to $\mathrm{Fe}^{3+}$ versus the other cations is that the valence electrons of
$\mathrm{Fe}^{3+}$ are $4 \mathrm{~s}^{2} 3 \mathrm{~d}^{5}$. The five $\mathrm{d}$ orbits are half-filled. The electrons of the complexes formed between $\mathrm{Fe}^{3+}$ and $\mathrm{H}$-CDs are easily transferred to the half-filled $3 \mathrm{~d}$ orbits of $\mathrm{Fe}^{3+}$, leading to a quenching of the fluorescence..$^{39}$

\section{Conclusions}

In summary, we have prepared hydroxyl functionalized carbon dots by monoesterification. The FTIR and XPS results show that the surface chemical composition of the CDs and H-CDs. The as-prepared H-CDs exhibit a narrower size distribution in the range of 1-4 nm (average of $2.0 \mathrm{~nm}$ ). The PL intensities of the H-CDs are greatly enhanced owing to the decrease in particle size and the change in the surface states. The fluorescence emission of the H-CDs can be quenched by $\mathrm{Fe}^{3+}$ both in aqueous solution and solid form with a detection limit as low as $2.56 \mathrm{nM}$.

\section{Acknowledgements}

This work was financially supported by National Natural Science Funds for Distinguished Young Scholars (No. 51425306), the National Key Research and Development Program of China (No. 2016YFA0202302), the State Key Program of National Natural Science Foundation of China (No. 51633007), and National Natural Science Foundation of China (No. 51373116, 51573125 and 513111129).

\section{Notes and references}

1 G. Cairo and A. Pietrangelo, Biochem. J., 2000, 353, 241-250.

2 R. R. Gaddam, D. Vasudevan, R. Narayan and K. V. S. N. Raju, RSC Adv., 2014, 4, 57137-57143.

3 S. Zhu, S. Tang, J. Zhang and B. Yang, Chem. Commun., 2012, 48, 4527-4539.

4 B. Liao, W. Wang, P. Long, B. Q. He, F. W. Li and Q. Q. Liu, RSC Adv., 2014, 4, 57683-57690.

5 J. Zhang, Y. Yuan, G. L. Liang and S. H. Yu, Adv. Sci., 2015, 2, 1500002.

6 A. Kumar, A. R. Chowdhuri, D. Laha, S. Chandra, P. Karmakar and S. K. Sahu, RSC Adv., 2016, 6, 58979-58987.

7 A. Tyagi, K. M. Tripathi, N. Singh, S. Choudhary and R. K. Gupta, $R S C A d v$., 2016, 6, 72423-72432.

8 K. G. Qu, J. S. Wang, J. S. Ren and X. G. Qu, Chem.-Eur. J., 2013, 19, 7243-7249.

9 A. Ananthanarayanan, X. W. Wang, P. Routh, B. Sana, S. R. Lim, D. H. Kim, K. H. Lim, J. Li and P. Chen, Adv. Funct. Mater., 2014, 24, 3021-3026.

10 Q. Xu, J. F. Wei, J. L. Wang, Y. Liu, N. Li, Y. S. Chen, C. Gao, W. W. Zhang and T. S. Sreeprased, RSC Adv., 2016, 6, 2874528750.

11 Q. S. Mei, K. Zhang, G. J. Guan, B. H. Liu, S. H. Wang and Z. P. Zhang, Chem. Commun., 2010, 46, 7319-7321.

12 S. J. Zhu, J. H. Zhang, S. J. Tang, C. Y. Qiao, L. Wang, H. Y. Wang, X. Liu, B. Li, Y. F. Li, W. L. Yu, X. F. Wang, H. C. Sun and B. Yang, Adv. Funct. Mater., 2012, 22, 47324740 . 
13 S. L. Hu, R. X. Tian, Y. G. Dong, J. L. Yang, J. Liu and Q. Chang, Nanoscale, 2013, 5, 11665-11671.

14 L. Q. Liu, Y. F. Li, L. Zhan, Y. Liu and C. Z. Huang, Sci. China: Chem., 2011, 54, 1342-1347.

15 S. L. Hu, R. X. Tian, L. L. Wu, Q. Zhao, J. L. Yang, J. Liu and S. R. Cao, Chem.-Asian J., 2013, 8, 1035-1041.

16 Q. Y. Xing, W. W. Pei and R. Q. Xu, J. Fundamental Organic Chemistry, Higher Education Press, Beijing, 3rd edn, 2005, pp. 243-245.

17 L. Bao, Z. L. Zhang, Z. Q. Tian, L. Zhang, C. Liu, Y. Lin, B. P. Qi and D. W. Pang, Adv. Mater., 2011, 23, 5801-5806.

18 S. Y. Lim, W. Shen and Z. Q. Gao, Chem. Soc. Rev., 2015, 44, 362-381.

19 Y. L. Wang, L. Wang, H. C. Zhang, Y. Liu, H. Y. Wang, Z. H. Kang and S. T. Lee, RSC Adv., 2013, 3, 3733-3738.

20 L. Song, Y. Y. Cui, C. F. Zhang, Z. B. Hu and X. F. Liu, RSC Adv., 2016, 6, 17704-17712.

21 H. B. Xu, S. H. Zhou, L. L. Xiao, S. Z. Li, T. Song, Y. Wang and Q. H. Yuan, Carbon, 2015, 87, 215-225.

22 H. Sharghi and M. H. Sarvari, Tetrahedron, 2003, 59, 36273633.

23 Z. A. Qiao, Y. F. Wang, Y. Gao, H. W. Li, T. Y. Dai, Y. L. Liu and Q. S. Huo, Chem. Commun., 2010, 46, 8812-8814.

24 S. Chandra, A. R. Chowdhuri, T. K. Mahto, A. Samui and S. K. Sahu, RSC Adv., 2016, 6, 72471-72478.

25 D. P. Kong, F. Y. Yan, Z. Y. Han, J. X. Xu, X. F. Guo and L. Chen, RSC Adv., 2016, 6, 67481-67487.

26 L. B. Tang, R. B. Ji, X. K. Cao, J. Y. Lin, H. X. Jiang, X. M. Li, K. S. Teng, C. M. Luk, S. J. Zeng, J. H. Hao and S. P. Lau, ACS Nano, 2012, 6, 5102-5110.
27 M. Sevilla and A. B. Fuertes, Chem.-Eur. J., 2009, 15, 41954203.

28 W. Feng, C. Q. Qin, Y. T. Shen, Y. Li, W. Luo, H. R. An and Y. Y. Feng, Sci. Rep., 2014, 4, 3777.

29 H. G. Zhao, D. F. Wang, M. Chaker and D. L. Ma, J. Phys. Chem. C, 2011, 115, 1620-1626.

30 W. J. Lin, K. L. Fritz, G. Guerin, G. R. Bardajee, S. Hinds, V. Sukhovatkin, E. H. Sargent, G. D. Scholes and M. A. Winnik, Langmuir, 2008, 24, 8215-8219.

31 X. Guo, C. F. Wang, Z. Y. Yu, C. Li and S. Chen, Chem. Commun., 2012, 48, 2692-2694.

32 S. N. Baker and G. A. Baker, Angew. Chem., Int. Ed., 2010, 49, 6726-6744.

33 Z. C. Yang, M. Wang, A. M. Yong, S. Y. Wong, X. H. Zhang, H. Tan, A. Y. Chang, X. Li and J. Wang, Chem. Commun., 2011, 47, 11615-11617.

34 B. B. Campos, R. Contreras-Cáceres, T. J. Bandosz, J. Jiménez-Jiménez, E. Rodríguez-Castellón, J. C. G. Esteves da Silva and M. Algarra, Carbon, 2016, 106, 171-178.

35 Z. C. Huang, Y. T. Shen, Y. Li, W. J. Zheng, Y. J. Xue, C. Q. Qin, B. Zhang, J. X. Hao and W. Feng, Nanoscale, 2014, 6, 13043-13052.

36 Q. Xu, P. Pu, J. G. Zhao, C. B. Dong, C. Gao, Y. S. Chen, J. R. Chen, Y. Liu and H. J. Zhou, J. Mater. Chem. A, 2015, 3, 542-546.

37 Y. L. Jiang, Q. R. Han, C. Jin, J. Zhang and B. X. Wang, Mater. Lett., 2015, 141, 366-368.

38 S. H. Li, Y. C. Li, J. Cao, J. Zhu, L. Z. Fan and X. H. Li, Anal. Chem., 2014, 86, 10201-10207.

39 Q. S. Mei, C. L. Jiang, G. J. Guan, K. Zhang, B. H. Liu, R. Y. Liu and Z. P. Zhang, Chem. Commun., 2012, 48, 7468-7470. 